\title{
Comparative Analysis of the Correlation Between Local Meteorological Elements and the Concentration of Airborne Fungi in the Beopjusa and Seonamsa Temples
}

\author{
Myoung Nam Kim I Bo A Lim I Jin Young Hong I Jeung Min Lee I Ji Hee Park ${ }^{1}$ I So Young Jeong \\ Restoration Technology Division, National Research Institute of Cultural Heritage, Daejeon, 34122, Korea \\ ${ }^{1}$ Corresponding Author: siesta44@korea.kr, +82-42-860-9435
}

\begin{abstract}
This study involved a comparative analysis of the correlation between meteorological elements and the concentration of airborne fungi(CFU) in relation to biological damage to two temples on piedmonts, which is a cultural heritage building. The work compared Beopjusa temple in Boeun(Chungcheongbuk-do Province) and Seonamsa temple in Suncheon(Jeollanam-do Province). Twelve meteorological elements and the CFU were measured and the Pearson correlation analysis was used to determine the degree of the relationship between them. The results showed that Beopjusa temple had high wind speed, high total horizontal radiation, high evaporation, and large number of days with precipitation. Seonamsa temple had high air temperature, high relative humidity, high dew point temperature, high sea level pressure, high precipitation, and high CFU. The CFU at Beopjusa temple did not have a linear correlation with meteorological elements, but at Seonamsa temple it was highly positive correlated with the number of days with precipitation, relative humidity, and precipitation, and was highly negative correlated with total horizontal radiation. In addition, Beopju and Seonamsa temple had a common linear relationship between factors not affected by the topographical conditions, and had a individual linear correlation between factors affected by the topographical conditions.
\end{abstract}

Key Words: Cultural heritage building, Biological damage, Meteorological elements, Concentration of airborne fungi(CFU), Correlation analysis

\section{INTRODUCTION}

The parts of the Beopjusa temple that are considered cultural heritage are the pagodas, stone lanterns, and halls, and those of the Seonamsa temple that are deemed heritage are the stone pagodas, stone bridges, stupas, and halls, most of which are either a national treasure or a treasure(Jogye Order of Korean Buddhism-Beopjusa temple, 2018; Taego Order of Korean Buddhism-Seonamsa temple, 2018) Beopjusa temple is located in a central inland region of Korea and Seonamsa temple is located in a southern coastal inland region of Korea. Since both temples are located on piedmonts, concerns for biological damage to their wood and stone structures are high.

For the wood structures, biodeterioration could be caused by fungi, bacteria, insects, and termites(Nilsson and
Rowell, 2012). For stone structures, biodeterioration could be caused by shrubs, lichens, mosses, herbaceous plants, algae, eumycetes, and bacteria(NRICH, 2011; 2014). This will be the case especially when microbiological conditions, such as temperature, moisture, and nutrients, are favorable (Wang et al., 2010). The presence and dispersal of airborne fungi depend on diverse parameters, including meteorological, biological, and topographical elements(De Linares et al., 2010), and exhibit daily and seasonal changes(Southworth, 1973; Hong et al., 2018). Moreover, the concentration and type of airborne fungi vary by region and season (MedrelaKuder, 2003; Oliveira et al., 2005) and are correlated with wind, temperature, humidity, precipitation, altitude, and vegetation of surrounding areas(Topbas et al., 2006).

Previous studies shed different lights on the concentration, diversity, and viability of airborne fungi. In a study that 
applied Pearson analysis to the correlation between the concentration of airborne fungi and meteorological elements (temperature, relative humidity, precipitation, total horizontal radiation, wind speed, wind direction, and surface temperature), the concentration of airborne fungi was shown to have a positive correlation with temperature and number of visitors, and a negative correlation with relative humidity and precipitation(Wang et al., 2010). In another study that used Spearman analysis and multiple regression analysis to investigate the correlation of the concentration of airborne fungi with meteorological elements and air pollutants $\left(\mathrm{NO}_{2}\right.$, $\mathrm{SO}_{2}$, and $\mathrm{PM}_{10}$ ), different results were obtained for different fungal species: aspergillus had a positive correlation with temperature while penicillium had a negative correlation, and the correlation between the concentration of fungi and air pollutants was unclear(Abdel Hameed et al., 2012). In a study that investigated the concentration of airborne fungi in particulate matter( $\left.\mathrm{PM}_{10}, \mathrm{PM}_{2.5}\right)$ and performed multiple regression analysis to determine the changes in airborne fungi in relation to air pollutants $\left(\mathrm{NO}_{2}, \mathrm{SO}_{2}, \mathrm{O}_{3}\right)$ and meteorological elements, the results indicated that the concentration of airborne fungi was strongly correlated with temperature, and $\mathrm{PM}_{10}$ and $\mathrm{PM}_{2.5}$ concentrations(Alghamdi et al., 2014).

Based on these findings, it may be concluded that the concentration of airborne fungi has a strong correlation with meteorological elements and particulate matter but a weak correlation with gaseous pollutants. Nonetheless, it is difficult to generalize and apply these findings because the correlation among different species of airborne fungi and individual meteorological elements is more complex than these study results make it seem and these results from different studies are inconsistent with each other. The inconsistencies may have been caused by the differences in climatic and seasonal conditions during each of those studies, in the specific meteorological elements taken into consideration, in the analytic tools used, and in the topographical conditions of each. Among these factors, the influence of topographical conditions can be assumed the largest, and thus, the differences in topography should not be ignored. As certain meteorological elements are greatly influenced by topographical conditions(Lee, 2015), it can be presumed that the meteorological characteristics reflect the unique topographical conditions of a given region.

This study monitored the local meteorological elements and the concentration of airborne microorganisms in the Beopjusa and Seonamsa temple under different topographical conditions, and used the Pearson correlation analysis to analyze and compare the correlation between meteorological elements and the concentration of airborne fungi at the two temples. This was done with the aim to derive the two temples' common properties and individual properties.

\section{METHODS}

\subsection{Monitoring sites}

Beopjusa temple is located at 405 Beopjusa-ro, Sokrisanmyeon, Boeun-gun, Chungcheongbuk-do in Korea. The building cultural heritages of the precinct are arranged along the NNE orientation from the Geumgangmun gate as they face the SSW direction. The meteorological elements were measured in the garden of Chongjiseonwon(latitude $36^{\circ} 32^{\prime} 36.40^{\prime \prime}$; longitude $127^{\circ} 50^{\prime} 03.25^{\prime \prime}$; altitude $352 \mathrm{~m}$ ), and the concentration of airborne fungi was sampled from the area in front of Myeongbu-jeon(Figure 1A, 1C, 1D). Seonamsa temple is located at 450 Seonamsa-gil, Seungju-eup, Suncheon-si, Jeollanam-do in Korea. The building cultural heritages of the precinct are arranged along the NNW orientation from the Iljumun gate as they face the SSE direction. The meteorological elements were measured in the area 70M north of Sanshin-gak(latitude 34 $59^{\prime} 51.90^{\prime \prime}$; longitude $127^{\circ} 19^{\prime} 47.68^{\prime \prime}$; altitude $265 \mathrm{~m}$ ), and the concentration of airborne fungi was sampled from the area in front of Josa-jeon(Figure 1B, 1E, 1F).

The topographical conditions around Beopjusa temple are as follows: altitude, $352 \mathrm{~m}$; relative height(the difference in elevation of the temple site from surrounding areas), $250 \mathrm{~m}$; aspect(topographic direction) and opening direction, facing SSW; small relief amount with flat slope; and distance from coastline $120 \mathrm{~km}$. The topographical conditions around Seonamsa temple are as follows: altitude, $265 \mathrm{~m}$; relative 
height, $235 \mathrm{~m}$; aspect and opening direction, facing SSE; large relief amount with steep slope; and distance from coastline, $20 \mathrm{~km}$.

\subsection{Measurement of meteorological elements}

Twelve meteorological elements(air temperature, relative humidity, dew point temperature, wind speed, wind direction, sea level pressure, precipitation, total horizontal radiation, evaporation, number of days with precipitation, mean UV Index, and maximum UV Index) were measured by installing an automatic weather station(Vantage Pro2 Plus, Davis Instrument, USA) and collecting the hourly observed values. This was done between June 2015 and April 2018 (Table 1). For wind direction, the Windrose program (WRPLOT View, Lakes Environmental, USA) was used.
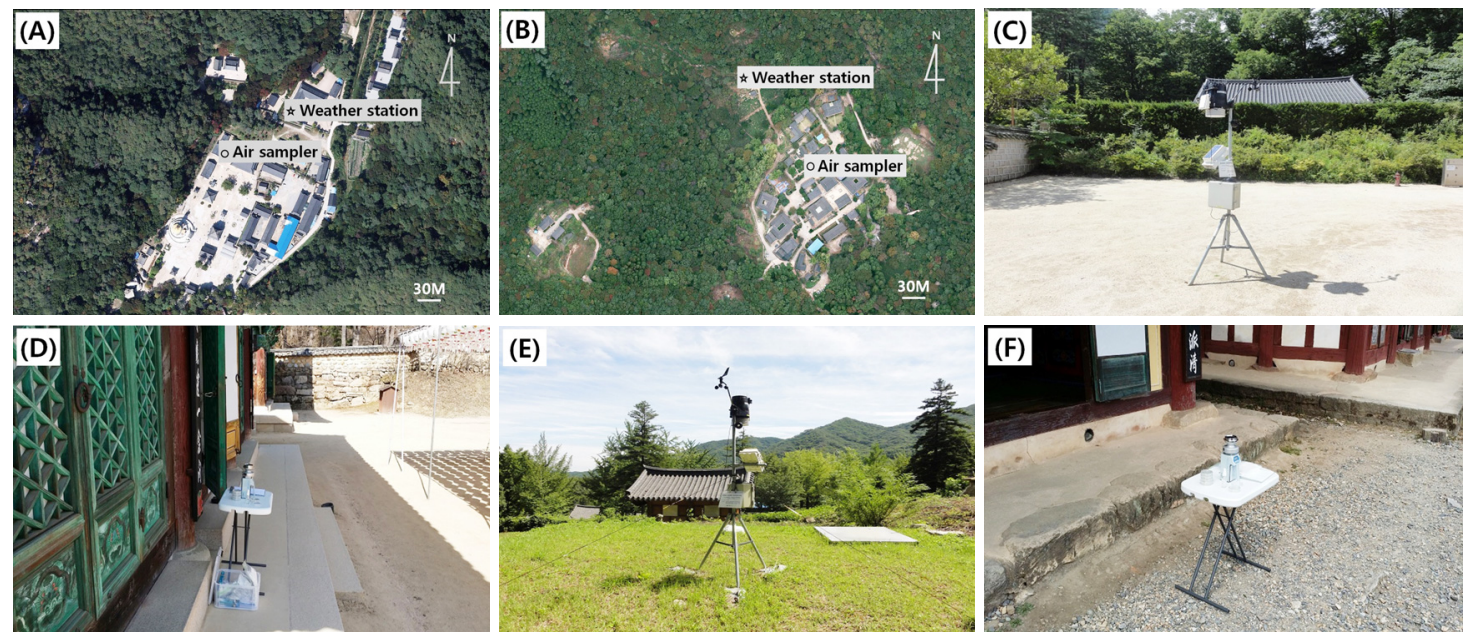

Figure 1. Meteorological elements and airborne fungi monitoring site. (A) Beopjusa temple, (B) Seonamsa temple,

(C) weather station(Beopjusa temple), (D) air sampler(Beopjusa temple), (E) weather station(Seonamsa temple),

(F) air sampler(Seonamsa temple).

Table 1. Monitoring meteorological elements and airborne fungi

\begin{tabular}{|c|c|c|c|}
\hline Items & Elements & Unit & Equipment \\
\hline \multirow{12}{*}{$\begin{array}{l}\text { Meteorological } \\
\text { element }\end{array}$} & Air temperature & ${ }^{\circ} \mathrm{C}$ & \multirow{12}{*}{$\begin{array}{c}\text { Vantage Pro2 Plus } \\
\text { (Davis Instrument, USA) }\end{array}$} \\
\hline & Relative humidity & $\%$ & \\
\hline & Dew point temperature & ${ }^{\circ} \mathrm{C}$ & \\
\hline & Wind speed & $\mathrm{m} / \mathrm{s}$ & \\
\hline & Wind direction & $\circ$ & \\
\hline & Sea level pressure & $\mathrm{hPa}$ & \\
\hline & Precipitation & $\mathrm{mm}$ & \\
\hline & Total horizontal fadiation & $\mathrm{MJ} / \mathrm{m}^{2}$ & \\
\hline & Evaporation & $\mathrm{mm}$ & \\
\hline & Number of days with precipitation & - & \\
\hline & Mean UV index & - & \\
\hline & Maximum UV index & - & \\
\hline Airborne fungi & Colony forming unit & $\mathrm{CFU} / \mathrm{m}^{3}$ & M Air T(Millipore, USA) \\
\hline
\end{tabular}




\subsection{Sampling of airborne fungi}

The airborne fungi in the outdoor air were sampled seasonally: spring(March-May), summer(June-August), fall (September-November), and winter(December-February); however, due to freezing of the culture media during winter when the air temperature dropped below zero, only the initial sampling was included in the analysis for winter. In the case of Beopjusa temple, the sampling was conducted twice in 2015(July 27, November 2), thrice in 2016(January 19, August 3, November 1), four times in 2017(March 21, April 25, July 25, October 31), and twice in 2018(March 27, April 27). In the case of Seonamsa temple, the sampling was conducted twice in 2015(July 28, November 3), four times in 2016(February 24, April 28, August 2, November 2), four times in 2017(March 22, April 26, July 26, November 2), and twice in 2018(March 28, April 23). The time of sampling was between 1 and $2 \mathrm{pm}$, and the method involved an air sampler(M Air T, Millipore, USA) equipped with plates containing PDA(Potato Dextrose Agar, Difco, USA), where $250 \mathrm{~L}(140 \mathrm{~L} / \mathrm{min})$ of air was collected thrice repeatedly. The plates with the collected air were cultured at $28^{\circ} \mathrm{C}$ for two to three days, after which the colonies were counted for calculating the $\mathrm{CFU}\left(\right.$ Colony Forming Unit $/ \mathrm{m}^{3}$ ) according to Equation (1).

$$
\begin{array}{r}
\mathrm{CFU} / \mathrm{m}^{3}=\text { Colony Forming Unit }(\mathrm{CFU}) / \\
{\left[\text { Air flow }(\mathrm{L}) \times 1000\left(\mathrm{~L} / \mathrm{m}^{3}\right)\right]}
\end{array}
$$

\subsection{Correlation analysis}

For analyzing the correlation between the meteorological elements and the concentration of airborne fungi, Pearson correlation analysis(IBM PASW Statistics 18) was applied to the airborne fungi sampled 11 times at Beopjusa temple and 12 times at Seonamsa temple between June 2015 and April 2018, and the observed values of the twelve meteorological elements on the days of sampling(Table 2).

\section{RESULTS}

\subsection{Monitoring local meteorological elements and the concentration of airborne fungi}

From the values observed seasonally through the monitoring period between June 2015 and April 2018, the

\begin{tabular}{|c|c|c|}
\hline \multirow{4}{*}{$r=\frac{\Sigma(x-\bar{x})(y-\bar{y})}{\sqrt{\Sigma(x-\bar{x})^{2} \times \Sigma(y-\bar{y})^{2}}}$} & \multicolumn{2}{|c|}{ Pearson correlation interpretation } \\
\hline & \multicolumn{2}{|r|}{$\begin{array}{c}*: \text { p-Value }<0.05 \\
* *: \text { p-Value }<0.01\end{array}$} \\
\hline & $-1.0 \sim-0.7$ & A strong negative linear relationship \\
\hline & $0.7 \sim 1.0$ & A strong positive linear relationship \\
\hline
\end{tabular}

Table 2. Person correlation coefficient (Left: Equation, Right: Pearson correlation interpretation)

\begin{tabular}{|c|c|c|c|c|c|c|c|c|c|c|c|}
\hline Site & $\begin{array}{l}\text { Season } \\
\text { (month) }\end{array}$ & $\begin{array}{c}\text { Air } \\
\text { temper- } \\
\text { ature }\left({ }^{\circ} \mathrm{C}\right)\end{array}$ & $\begin{array}{c}\text { Relative } \\
\text { humidity } \\
(\%)\end{array}$ & $\begin{array}{c}\text { Dew } \\
\text { point } \\
\text { temper- } \\
\text { ature }\left({ }^{\circ} \mathrm{C}\right)\end{array}$ & $\begin{array}{l}\text { Wind } \\
\text { speed } \\
(\mathrm{m} / \mathrm{s})\end{array}$ & $\begin{array}{c}\text { Sea } \\
\text { level } \\
\text { pressure } \\
(\mathrm{hPa})\end{array}$ & $\begin{array}{l}\text { Precipit- } \\
\text { ation } \\
(\mathrm{mm})\end{array}$ & $\begin{array}{c}\text { Total } \\
\text { horizontal } \\
\text { radiation } \\
\left(\mathrm{MJ} / \mathrm{m}^{2}\right)\end{array}$ & $\begin{array}{c}\text { Evapor- } \\
\text { ation } \\
(\mathrm{mm})\end{array}$ & $\begin{array}{l}\text { Number of } \\
\text { days with } \\
\text { precipit- } \\
\text { ation }\end{array}$ & $\begin{array}{c}\text { Wind } \\
\text { direction } \\
\left({ }^{\circ}\right)\end{array}$ \\
\hline \multirow{5}{*}{$\begin{array}{l}\text { Beopjusa } \\
\text { temple }\end{array}$} & Annual & 11.1 & 76.2 & 6.4 & 0.5 & 1015.3 & 1011.8 & 4385.7 & 774.8 & 129.7 & 112.5 \\
\hline & Spring(3-5) & 11.1 & 68.7 & 4.4 & 0.6 & 1014.2 & 201.0 & 1484.6 & 265.6 & 27.7 & 112.5 \\
\hline & Summer(6-8) & 22.8 & 81.3 & 19.0 & 0.4 & 1006.4 & 552.1 & 1401.7 & 278.9 & 40.3 & 270 \\
\hline & Fall(9-11) & 12.2 & 81.2 & 8.5 & 0.5 & 1017.6 & 186.3 & 835.6 & 149.6 & 33.3 & 270 \\
\hline & Winter(12-1) & -1.8 & 73.7 & -6.4 & 0.5 & 1022.9 & 72.4 & 663.8 & 80.6 & 28.3 & 112.5 \\
\hline \multirow{5}{*}{$\begin{array}{l}\text { Seonamsa } \\
\text { temple }\end{array}$} & Annual & 12.8 & 76.6 & 8.2 & 0.4 & 1015.9 & 1232.2 & 4355.2 & 765.9 & 126.0 & 135 \\
\hline & Spring(3-5) & 12.6 & 68.9 & 6.1 & 0.5 & 1015.1 & 331.7 & 1441.4 & 259.1 & 31.7 & 135 \\
\hline & Summer(6-8) & 22.9 & 86.4 & 20.2 & 0.2 & 1007.4 & 450.2 & 1259.3 & 237.3 & 38.3 & 135 \\
\hline & Fall(9-11) & 14.3 & 82.1 & 10.9 & 0.3 & 1017.8 & 332.8 & 912.2 & 160.2 & 29.7 & 135 \\
\hline & Winter(12-1) & 1.3 & 69.0 & -4.4 & 0.7 & 1023.3 & 117.5 & 742.2 & 109.3 & 26.3 & 112.5 \\
\hline
\end{tabular}

Table 3. Results of meteorological element observations(June 2015-April 2018) 
annual mean and seasonal mean values were obtained for comparison between the meteorological characteristics of Beopjusa temple and Seonamsa temple(Table 3). The annual mean air temperature at Beopjusa temple was $11.1^{\circ} \mathrm{C}$ and Seonamsa temple was $12.8^{\circ} \mathrm{C}$, indicating a $1.7^{\circ} \mathrm{C}$ higher air temperature at Seonamsa temple. The annual mean humidity at Beopjusa temple was $76.2 \%$ and Seonamsa temple was $76.6 \%$, indicating a $0.4 \%$ higher humidity at Seonamsa temple. The annual mean dew point temperature at Beopjusa temple was $6.4^{\circ} \mathrm{C}$ and Seonamsa temple was $8.2^{\circ} \mathrm{C}$, indicating a $1.8^{\circ} \mathrm{C}$ higher temperature at Seonamsa temple. The annual mean wind speed at Beopjusa temple was $0.5 \mathrm{~m} / \mathrm{s}$ and Seonamsa temple was $0.4 \mathrm{~m} / \mathrm{s}$, indicating a $0.1 \mathrm{~m} / \mathrm{s}$ faster wind at Beopjusa temple. The average sea level pressure at Beopjusa temple was $1015.3 \mathrm{hPa}$ and
Seonamsa temple was $1015.9 \mathrm{hPa}$, showing a $0.6 \mathrm{hPa}$ higher pressure at Seonamsa temple. The precipitation at Beopjusa temple was $1011.8 \mathrm{~mm}$ and Seonamsa temple was $1232.2 \mathrm{~mm}$, indicating that Seonamsa temple received $220.4 \mathrm{~mm}$ more rainfall. The total horizontal radiation at Beopjusa temple was $4385.7 \mathrm{MJ} / \mathrm{m}^{2}$ and Seonamsa temple was $4355.2 \mathrm{MJ} / \mathrm{m}^{2}$, indicating that Beopjusa temple received $30.5 \mathrm{MJ} / \mathrm{m}^{2}$ more radiation. The total evaporation at Beopjusa temple was $774.8 \mathrm{~mm}$ and Seonamsa temple was $765.9 \mathrm{~mm}$, indicating $8.9 \mathrm{~mm}$ more evaporation at Beopjusa temple. For number of days with precipitation, Beopjusa temple had 129.7 days of rainfall on average while Seonamsa temple had 126.0 days, indicating 3.7 days more rainfall at Beopjusa temple. The main wind direction at Beopjusa temple was $112.5^{\circ}$ (ESE wind) and Seonamsa

Table 4. Meteorological element observations(daily) and the concentration of airborne fungi at Beopjusa and Seonamsa temple

\begin{tabular}{|c|c|c|c|c|c|c|c|c|c|c|c|c|c|c|}
\hline Site & $\begin{array}{c}\text { Measuring } \\
\text { date }\end{array}$ & $\begin{array}{l}\text { Air } \\
\text { temper- } \\
\text { ature } \\
\left({ }^{\circ} \mathrm{C}\right)\end{array}$ & $\begin{array}{c}\text { Relative } \\
\text { humidity } \\
(\%)\end{array}$ & $\begin{array}{c}\text { Dew } \\
\text { point } \\
\text { temper- } \\
\text { ature } \\
\left({ }^{\circ} \mathrm{C}\right)\end{array}$ & $\begin{array}{l}\text { Wind } \\
\text { speed } \\
(\mathrm{m} / \mathrm{s})\end{array}$ & $\begin{array}{c}\text { Sea } \\
\text { level } \\
\text { pressure } \\
(\mathrm{hPa})\end{array}$ & $\begin{array}{l}\text { Precipit- } \\
\text { ation } \\
(\mathrm{mm})\end{array}$ & $\begin{array}{c}\text { Total } \\
\text { horizontal } \\
\text { radiation } \\
\left(\mathrm{MJ} / \mathrm{m}^{2}\right)\end{array}$ & $\begin{array}{c}\text { Evapor- } \\
\text { ation } \\
(\mathrm{mm})\end{array}$ & $\begin{array}{l}\text { Number } \\
\text { of days } \\
\text { with } \\
\text { precipit- } \\
\text { ation }\end{array}$ & $\begin{array}{c}\text { Mean } \\
\text { UV } \\
\text { index }\end{array}$ & $\begin{array}{c}\text { Maxim- } \\
\text { um UV } \\
\text { index }\end{array}$ & $\begin{array}{c}\text { Wind } \\
\text { direction } \\
\left(^{\circ}\right)\end{array}$ & $\mathrm{CFU} / \mathrm{m}^{2}$ \\
\hline \multirow{11}{*}{$\begin{array}{c}\text { Beopjusa } \\
\text { temple }\end{array}$} & $07-27-2015$ & 24.1 & 90.9 & 22.5 & 0.2 & 1007.6 & 1.0 & 8.4 & 1.6 & 1 & 1.8 & 4.6 & 135 & 96 \\
\hline & $11-02-2015$ & 7.6 & 74.1 & 2.9 & 0.5 & 1022.6 & 0.0 & 9.8 & 1.5 & 0 & 1.5 & 2.3 & 112.5 & 249.3 \\
\hline & 01-19-2016 & -10.7 & 77.7 & -13.9 & 1.2 & 1019.1 & 0.0 & 4.3 & 0.3 & 0 & 0.8 & 1.1 & 112.5 & 200 \\
\hline & 08-03-2016 & 24.8 & 88.3 & 22.6 & 0.2 & 1008.0 & 0.0 & 16.2 & 3.0 & 0 & 3.5 & 7.0 & 0 & 1002 \\
\hline & $11-01-2016$ & 0.4 & 65.1 & -5.5 & 0.9 & 1027.6 & 0.0 & 2.2 & 0.4 & 0 & 1.1 & 1.6 & 112.5 & 284.7 \\
\hline & $03-21-2017$ & 5.9 & 65.5 & -0.9 & 0.6 & 1014.2 & 0.0 & 17.0 & 2.6 & 0 & 2.1 & 3.8 & 112.5 & 117.3 \\
\hline & $04-25-2017$ & 14.2 & 70.8 & 8.5 & 0.5 & 1017.4 & 0.0 & 15.5 & 2.8 & 0 & 2.2 & 4.4 & 135 & 178 \\
\hline & $07-25-2017$ & 25.5 & 90.4 & 23.7 & 0.5 & 1002.1 & 44.4 & 13.6 & 2.8 & 1 & 3.2 & 5.4 & 270 & 256.7 \\
\hline & $10-31-2017$ & 2.5 & 73.1 & -2.9 & 0.3 & 1025.7 & 0.0 & 8.7 & 1.2 & 0 & 2.3 & 3.5 & 135 & 390 \\
\hline & 03-27-2018 & 7.1 & 82.8 & 3.8 & 0.2 & 1017.9 & 0.0 & 8.0 & 1.3 & 0 & 2.1 & 4.2 & 135 & 96 \\
\hline & 04-27-2018 & 12.7 & 68.9 & 6.2 & 0.4 & 1012.9 & 0.0 & 21.3 & 3.7 & 0 & 2.6 & 4.6 & 112.5 & 200 \\
\hline \multirow{12}{*}{$\begin{array}{l}\text { Seonamsa } \\
\text { temple }\end{array}$} & $07-28-2015$ & 25.6 & 90.0 & 23.7 & 0.2 & 1008.6 & 0.0 & 11.5 & 2.3 & 0 & 2.4 & 4.8 & 112.5 & 128 \\
\hline & 11-03-2015 & 12.0 & 71.0 & 6.3 & 0.5 & 1028.0 & 0.0 & 13.6 & 2.4 & 0 & 1.8 & 3.1 & 157.5 & 196 \\
\hline & 02-24-2016 & -2.0 & 57.4 & -9.4 & 1.3 & 1031.5 & 0.0 & 15.3 & 2.0 & 0 & 1.5 & 2.7 & 135 & 24 \\
\hline & 04-28-2016 & 12.8 & 93.5 & 11.7 & 0.1 & 1007.0 & 4.2 & 8.6 & 1.3 & 1 & 1.3 & 2.2 & 0 & 652 \\
\hline & 08-02-2016 & 25.9 & 90.8 & 24.2 & 0.1 & 1008.1 & 0.8 & 9.5 & 1.8 & 1 & 1.7 & 3.0 & 0 & 668 \\
\hline & $11-02-2016$ & 3.9 & 60.3 & -3.4 & 1.0 & 1027.9 & 0.0 & 6.7 & 1.1 & 0 & 1.7 & 2.9 & 180 & 220 \\
\hline & 03-22-2017 & 6.3 & 56.2 & -3.0 & 0.4 & 1020.0 & 0.2 & 14.2 & 2.3 & 1 & 2.3 & 4.0 & 135 & 92 \\
\hline & 04-26-2017 & 13.6 & 72.4 & 8.1 & 0.3 & 1014.7 & 2.2 & 9.1 & 1.8 & 1 & 1.6 & 3.1 & 225 & 598 \\
\hline & 07-26-2017 & 25.0 & 83.0 & 21.7 & 0.0 & 1006.6 & 0.0 & 20.0 & 3.7 & 0 & 3.9 & 8.0 & 0 & 259.3 \\
\hline & $11-02-2017$ & 11.0 & 85.8 & 8.4 & 0.1 & 1018.8 & 0.0 & 7.3 & 1.1 & 0 & 1.8 & 2.8 & 0 & 174 \\
\hline & 03-28-2018 & 14.4 & 58.5 & 5.6 & 0.5 & 1016.8 & 0.0 & 20.7 & 4.0 & 0 & 2.5 & 4.6 & 90 & 198 \\
\hline & 04-23-2018 & 11.8 & 98.0 & 11.5 & 0.2 & 1013.8 & 40.0 & 0.8 & 0.1 & 1 & 0.0 & 0.0 & 0 & 854 \\
\hline
\end{tabular}


temple was $135^{\circ}$ (SE wind). To sum up, Beopjusa temple exhibited higher wind speed, total horizontal radiation, evaporation, and number of days with precipitation; while Seonamsa temple exhibited higher air temperature, humidity, dew point temperature, sea level pressure, and precipitation. Seasonally, the mean humidity was higher at Beopjusa temple during winter but higher at Seonamsa temple during spring, summer, and fall. The mean wind speed was higher at Seonamsa temple during winter. The precipitation was greater at Beopjusa temple mostly during summer, whereas at Seonamsa temple large precipitation was dispersed throughout spring and fall with the largest precipitation occurring during summer. The total horizontal radiation was higher at Beopjusa temple during spring and summer, but higher at Seonamsa temple during fall and winter. For number of days with precipitation, Beopjusa temple had more days with rain mostly during summer while Seonamsa temple had more days with rain during spring, summer, and fall. The wind direction changed from east-southeast in winter and spring to west in summer and fall at Beopjusa temple; while at Seonamsa temple, only the southeast wind blew throughout the year. Table 4 summarizes the concentration of the airborne fungi and the meteorological elements measured on the day they were sampled and, and Table 5 presents the annual and seasonal values. When the annual mean values for the daily meteorological elements were compared with those for the entire monitoring period(given in Table 3), both Beopjusa and Seonamsa temples showed values reflecting the annual trend with respect to air temperature, dew point temperature, wind speed, sea level pressure, precipitation, and wind direction; while with respect to humidity, total horizontal radiation, evaporation, and number of days with precipitation, the influence of the daily meteorological phenomena was stronger so that the values did not reflect the annual trend. The concentration of airborne fungi was found to be higher at Seonamsa temple: $279.1 \mathrm{CFU} / \mathrm{m}^{3}$ at Beopjusa temple and 338.6 CFU $/ \mathrm{m}^{3}$ at Seonamsa temple. At Beopjusa temple, the concentration was the highest in summer, followed by fall. At Seonamsa temple, the highest concentration was observed in spring, followed by summer. Both Beopjusa and Seonamsa temples showed increased concentration of airborne fungi during summer with high air temperature, humidity, dew point temperature, precipitation, and number of days with precipitation, but slow wind speed. Notably, the concentration of airborne fungi was the highest during

Table 5. Meteorological element observations(annual and seasonal) and the concentration of airborne fungi at Beopjusa and Seonamsa temple

\begin{tabular}{|c|c|c|c|c|c|c|c|c|c|c|c|c|c|c|}
\hline Site & $\begin{array}{l}\text { Season } \\
\text { (month) }\end{array}$ & $\begin{array}{l}\text { Air } \\
\text { temper- } \\
\text { ature } \\
\left({ }^{\circ} \mathrm{C}\right)\end{array}$ & $\begin{array}{c}\text { Relative } \\
\text { humidity } \\
(\%)\end{array}$ & $\begin{array}{c}\text { Dew } \\
\text { point } \\
\text { temper- } \\
\text { ature } \\
\left({ }^{\circ} \mathrm{C}\right)\end{array}$ & $\begin{array}{l}\text { Wind } \\
\text { speed } \\
(\mathrm{m} / \mathrm{s})\end{array}$ & $\begin{array}{c}\text { Sea } \\
\text { level } \\
\text { pressure } \\
(\mathrm{hPa})\end{array}$ & $\begin{array}{c}\text { Precipit- } \\
\text { ation } \\
(\mathrm{mm})\end{array}$ & $\begin{array}{c}\text { Total } \\
\text { horizontal } \\
\text { radiation } \\
\left(\mathrm{MJ} / \mathrm{m}^{2}\right)\end{array}$ & $\begin{array}{c}\text { Evapor- } \\
\text { ation } \\
(\mathrm{mm})\end{array}$ & $\begin{array}{l}\text { Number } \\
\text { of days } \\
\text { with } \\
\text { precipit- } \\
\text { ation }\end{array}$ & $\begin{array}{c}\text { Mean } \\
\text { UV } \\
\text { index }\end{array}$ & $\begin{array}{l}\text { Maxim- } \\
\text { um UV } \\
\text { index }\end{array}$ & $\begin{array}{c}\text { Wind } \\
\text { direction } \\
\left(^{\circ}\right)\end{array}$ & $\mathrm{CFU} / \mathrm{m}^{2}$ \\
\hline \multirow{5}{*}{$\begin{array}{l}\text { Beopjusa } \\
\text { temple }\end{array}$} & Annual & 10.4 & 77.1 & 6.1 & 0.5 & 1015.9 & 45.4 & 125.0 & 21.2 & 2.0 & 2.1 & 7.0 & 112.5 & 279.1 \\
\hline & $\begin{array}{c}\text { Spring } \\
(3-5)\end{array}$ & 10.0 & 72.0 & 4.4 & 0.4 & 1015.6 & 0.0 & 61.8 & 10.4 & 0.0 & 2.3 & 4.3 & 112.5 & 147.8 \\
\hline & $\begin{array}{c}\text { Summer } \\
(6-8)\end{array}$ & 24.8 & 89.9 & 22.9 & 0.3 & 1005.9 & 45.4 & 38.2 & 7.4 & 2.0 & 2.8 & 5.7 & 270.0 & 451.6 \\
\hline & $\begin{array}{c}\text { Fall } \\
(9-11)\end{array}$ & 3.5 & 70.8 & -1.8 & 0.6 & 1025.3 & 0.0 & 20.7 & 3.1 & 0.0 & 1.6 & 2.5 & 112.5 & 308.0 \\
\hline & $\begin{array}{l}\text { Winter } \\
(12-1)\end{array}$ & -10.7 & 77.7 & -13.9 & 1.2 & 1019.1 & 0.0 & 4.3 & 0.3 & 0.0 & 0.8 & 1.1 & 112.5 & 200.0 \\
\hline \multirow{5}{*}{$\begin{array}{l}\text { Seonamsa } \\
\text { temple }\end{array}$} & Annual & 13.4 & 76.4 & 8.8 & 0.4 & 1016.8 & 47.4 & 137.3 & 23.9 & 5.0 & 1.9 & 3.4 & 135.0 & 338.6 \\
\hline & $\begin{array}{c}\text { Spring } \\
(3-5)\end{array}$ & 11.8 & 75.7 & 6.8 & 0.3 & 1014.5 & 46.6 & 53.4 & 9.5 & 4.0 & 1.5 & 2.8 & - & 478.8 \\
\hline & $\begin{array}{l}\text { Summer } \\
(6-8)\end{array}$ & 25.5 & 87.9 & 23.2 & 0.1 & 1007.8 & 0.8 & 41.0 & 7.8 & 1.0 & 2.7 & 5.3 & 112.5 & 351.8 \\
\hline & $\begin{array}{c}\text { Fall } \\
(9-11)\end{array}$ & 9.0 & 72.4 & 3.8 & 0.5 & 1024.9 & 0.0 & 27.6 & 4.6 & 0.0 & 1.8 & 2.9 & - & 196.7 \\
\hline & $\begin{array}{l}\text { Winter } \\
(12-1)\end{array}$ & -2.0 & 57.4 & -9.4 & 1.3 & 1031.5 & 0.0 & 15.3 & 2.0 & 0.0 & 1.5 & 2.7 & 135.0 & 24.0 \\
\hline
\end{tabular}


Table 6. Correlation analysis between meteorological elements and the concentration of airborne fungi at Beopjusa temple ( $\square$ : Common properties, $\square$ : Individual properties)

\begin{tabular}{|c|c|c|c|c|c|c|c|c|c|c|c|c|c|}
\hline $\begin{array}{l}\text { Beopjusa } \\
\text { temple }\end{array}$ & $\begin{array}{c}\text { Air } \\
\text { temperature } \\
\left({ }^{\circ} \mathrm{C}\right)\end{array}$ & $\begin{array}{c}\text { Relative } \\
\text { humidity } \\
(\%)\end{array}$ & $\begin{array}{c}\text { Dew } \\
\text { point } \\
\text { temper- } \\
\text { ature } \\
\left({ }^{\circ} \mathrm{C}\right)\end{array}$ & $\begin{array}{l}\text { Wind } \\
\text { speed } \\
(\mathrm{m} / \mathrm{s})\end{array}$ & $\begin{array}{c}\text { Sea level } \\
\text { pressure } \\
(\mathrm{hPa})\end{array}$ & $\begin{array}{l}\text { Precipit- } \\
\text { ation } \\
(\mathrm{mm})\end{array}$ & $\begin{array}{c}\text { Total } \\
\text { hori- } \\
\text { zontal } \\
\text { radiation } \\
\left(\mathrm{MJ} / \mathrm{m}^{2}\right)\end{array}$ & $\begin{array}{c}\text { Evapora } \\
\text { tion } \\
(\mathrm{mm})\end{array}$ & $\begin{array}{l}\text { Number } \\
\text { of days } \\
\text { with } \\
\text { precipit- } \\
\text { ation }\end{array}$ & $\begin{array}{l}\text { Maxim- } \\
\text { um UV } \\
\text { index }\end{array}$ & $\begin{array}{l}\text { Maxim- } \\
\text { um UV } \\
\text { index }\end{array}$ & $\begin{array}{c}\text { Wind } \\
\text { direction } \\
\left({ }^{\circ}\right)\end{array}$ & $\mathrm{CFU} / \mathrm{m}^{3}$ \\
\hline $\begin{array}{l}\text { Air temperature } \\
\left({ }^{\circ} \mathrm{C}\right)\end{array}$ & 1 & & & & & & & & & & & & \\
\hline $\begin{array}{c}\text { Relative } \\
\text { humidity (\%) }\end{array}$ & $.627^{*}$ & 1 & & & & & & & & & & & \\
\hline $\begin{array}{c}\text { Dew point } \\
\text { temperature } \\
\left({ }^{\circ} \mathrm{C}\right)\end{array}$ & $.991 * *$ & $.725 *$ & 1 & & & & & & & & & & \\
\hline $\begin{array}{l}\text { Wind speed } \\
(\mathrm{m} / \mathrm{s})\end{array}$ & $-.712^{*}$ & -.434 & $-.689 *$ & 1 & & & & & & & & & \\
\hline $\begin{array}{c}\text { Sea level } \\
\text { pressure }(\mathrm{hPa})\end{array}$ & $-.796 * *$ & $-.702^{*}$ & $-.823^{* *}$ & .377 & 1 & & & & & & & & \\
\hline $\begin{array}{l}\text { Precipitation } \\
(\mathrm{mm})\end{array}$ & .452 & .469 & .482 & -.007 & -.587 & 1 & & & & & & & \\
\hline $\begin{array}{c}\text { Total } \\
\text { horizontal } \\
\text { radiation } \\
\left(\mathrm{MJ} / \mathrm{m}^{2}\right)\end{array}$ & .534 & -.050 & .452 & -.442 & -.543 & .124 & 1 & & & & & & \\
\hline $\begin{array}{l}\text { Evaporation } \\
(\mathrm{mm})\end{array}$ & $.687^{*}$ & .085 & $.613 *$ & -.501 & $-.647 *$ & .258 & $.974 * *$ & 1 & & & & & \\
\hline $\begin{array}{l}\text { Number of days } \\
\text { with } \\
\text { precipitation }\end{array}$ & $.628^{*}$ & $.695^{*}$ & $.678^{*}$ & -.237 & $-.691^{*}$ & $.687^{*}$ & -.031 & .121 & 1 & & & & \\
\hline $\begin{array}{l}\text { Mean UV } \\
\text { index }\end{array}$ & $.777 * *$ & .428 & $.750 * *$ & $-.680 *$ & $-.668^{*}$ & .443 & $.723 *$ & $.803 * *$ & .238 & 1 & & & \\
\hline $\begin{array}{l}\text { Maximum UV } \\
\text { index }\end{array}$ & $.876^{* *}$ & .550 & $.860 * *$ & $-.771 * *$ & $-.756^{* * *}$ & .303 & $.686^{*}$ & $.782 * *$ & .330 & $.939 * *$ & 1 & & \\
\hline $\begin{array}{c}\text { Wind direction } \\
\left({ }^{\circ}\right)\end{array}$ & .129 & .176 & .142 & .082 & -.241 & $.786 * *$ & -.084 & -.002 & $.625^{*}$ & .029 & -.079 & 1 & \\
\hline $\mathrm{CFU} / \mathrm{m}^{3}$ & .315 & .280 & .330 & -.238 & -.158 & -.035 & .185 & .226 & -.199 & .544 & .493 & -.591 & 1 \\
\hline
\end{tabular}

the season with the largest precipitation, and it was seen to be affected by the daily meteorological phenomena.

\subsection{Correlation analysis between local meteorological elements and the concentration of airborne fungi}

From analyzing the correlation between meteorological elements and the concentration of airborne fungi, no linear correlation between the two was found for Beopjusa temple; while for Seonamsa temple, concentration of airborne fungi displayed a high correlation with number of days with precipitation, $0.752(\mathrm{p}<0.01)$; relative humidity, $0.666(\mathrm{p}<0.05)$; precipitation, $0.657(\mathrm{p}<0.05)$; and total horizontal radiation, $-0.614(\mathrm{p}<0.05)$. Both Beopjusa and Seonamsa temples showed strong linear correlations between relative humidity and air temperature; dew point temperature and air temperature and relative humidity; wind speed and air temperature and dew point temperature; sea level pressure and air temperature, relative humidity, and dew point temperature; evaporation and total horizontal radiation; mean UV Index and total horizontal radiation and evaporation; and max UV Index and total horizontal radiation, evaporation, and mean UV Index (Common properties are marked as $\square$ ). Independently, Beopjusa temple showed strong linear correlations between evaporation and air temperature, dew point temperature, and sea level pressure; number of days with precipitation and air temperature, relative humidity, dew point temperature, sea level pressure, and precipitation; mean UV Index and air temperature, dew point temperature, wind speed, and sea 
Table 7. Correlation analysis between meteorological elements and the concentration of airborne fungi at Seonamsa temple ( $\square$ : Common properties, $\square$ : Individual properties)

\begin{tabular}{|c|c|c|c|c|c|c|c|c|c|c|c|c|c|}
\hline $\begin{array}{l}\text { Seonamsa } \\
\text { temple }\end{array}$ & $\begin{array}{c}\text { Air } \\
\text { temperature } \\
\left({ }^{\circ} \mathrm{C}\right)\end{array}$ & $\begin{array}{c}\text { Relative } \\
\text { humidity } \\
(\%)\end{array}$ & $\begin{array}{c}\text { Dew } \\
\text { point } \\
\text { temper- } \\
\text { ature } \\
\left({ }^{\circ} \mathrm{C}\right)\end{array}$ & $\begin{array}{l}\text { Wind } \\
\text { speed } \\
(\mathrm{m} / \mathrm{s})\end{array}$ & $\begin{array}{l}\text { Sea Level } \\
\text { Pressure } \\
(\mathrm{hPa})\end{array}$ & $\begin{array}{l}\text { Precipit- } \\
\text { ation } \\
(\mathrm{mm})\end{array}$ & $\begin{array}{c}\text { Total } \\
\text { hori- } \\
\text { zontal } \\
\text { radiation } \\
\left(\mathrm{MJ} / \mathrm{m}^{2}\right)\end{array}$ & $\begin{array}{c}\text { Evapor- } \\
\text { ation } \\
(\mathrm{mm})\end{array}$ & $\begin{array}{l}\text { Number } \\
\text { of days } \\
\text { with } \\
\text { precipit- } \\
\text { ation }\end{array}$ & $\begin{array}{l}\text { Mean UV } \\
\text { index }\end{array}$ & $\begin{array}{l}\text { Maxim- } \\
\text { um UV } \\
\text { index }\end{array}$ & $\begin{array}{c}\text { Wind } \\
\text { direction } \\
\left({ }^{\circ}\right)\end{array}$ & $\mathrm{CFU} / \mathrm{m}^{3}$ \\
\hline $\begin{array}{c}\text { Air } \\
\text { temperature } \\
\left({ }^{\circ} \mathrm{C}\right)\end{array}$ & 1 & & & & & & & & & & & & \\
\hline $\begin{array}{c}\text { Relative. } \\
\text { humidity (\%) }\end{array}$ & $.626^{*}$ & 1 & & & & & & & & & & & \\
\hline $\begin{array}{c}\text { Dew Point } \\
\text { temperature } \\
\left({ }^{\circ} \mathrm{C}\right)\end{array}$ & $.971^{* *}$ & $.794 * *$ & 1 & & & & & & & & & & \\
\hline $\begin{array}{l}\text { Wind speed } \\
(\mathrm{m} / \mathrm{s})\end{array}$ & $-.777 * *$ & $-.749^{* *}$ & $-.819^{* *}$ & 1 & & & & & & & & & \\
\hline $\begin{array}{c}\text { Sea level } \\
\text { pressure }(\mathrm{hPa})\end{array}$ & $-.831 * *$ & $-.731 * *$ & $-.864 * *$ & $.860^{* *}$ & 1 & & & & & & & & \\
\hline $\begin{array}{l}\text { Precipitation } \\
(\mathrm{mm})\end{array}$ & -.051 & .476 & .096 & -.188 & -.158 & 1 & & & & & & & \\
\hline $\begin{array}{c}\text { Total } \\
\text { horizontal } \\
\text { radiation } \\
\left(\mathrm{MJ} / \mathrm{m}^{2}\right) \\
\end{array}$ & .140 & -.521 & -.048 & .137 & .041 & $-.620^{*}$ & 1 & & & & & & \\
\hline $\begin{array}{c}\text { Evaporation } \\
(\mathrm{mm})\end{array}$ & .330 & -.416 & .129 & -.021 & -.110 & $-.578^{*}$ & $.966^{* *}$ & 1 & & & & & \\
\hline $\begin{array}{c}\text { Number of days } \\
\text { with } \\
\text { precipitation }\end{array}$ & .073 & .327 & .141 & -.384 & -.415 & .427 & -.466 & -.431 & 1 & & & & \\
\hline $\begin{array}{l}\text { Mean UV } \\
\text { index }\end{array}$ & .419 & -.265 & .249 & -.166 & -.215 & $-.684^{*}$ & $.816^{* * *}$ & $.848^{* *}$ & -.481 & 1 & & & \\
\hline$\underset{\text { index }}{\text { Maximum UV }}$ & .476 & -.201 & .314 & -.193 & -.280 & $-.604^{*}$ & $.801 * *$ & $.845^{* *}$ & -.454 & $.989 * *$ & 1 & & \\
\hline $\begin{array}{c}\text { Wind direction } \\
\left({ }^{\circ}\right)\end{array}$ & -.431 & $-.704 *$ & -.548 & $.605^{*}$ & $.599 *$ & -.342 & .133 & .120 & -.152 & .020 & .007 & 1 & \\
\hline $\mathrm{CFU} / \mathrm{m}^{3}$ & .299 & $.666^{*}$ & .429 & -.488 & -.537 & $.657^{*}$ & $-.614^{*}$ & -.508 & $.752 * *$ & -.574 & -.506 & -.389 & 1 \\
\hline
\end{tabular}

*: Correlation is significant at the 0.05 level(2-tailed).

$* *$ : Correlation is significant at the 0.01 level(2-tailed).

level pressure; max UV Index and air temperature, dew point temperature, wind speed, and sea level pressure; and wind direction and precipitation and number of days with precipitation. Seonamsa temple showed strong linear correlations between wind speed and relative humidity; sea level pressure and wind speed; total horizontal radiation and precipitation; evaporation and precipitation; mean UV Index and precipitation; maximum UV Index and precipitation; wind direction and relative humidity, wind speed, and sea level pressure; and concentration of airborne fungi and relative humidity, precipitation, total horizontal radiation, and number of days with precipitation(Individual properties are marked as $\square)($ Tables 6,7$)$.

\section{DISCUSSION AND CONCLUSION}

For Beopjusa temple, no linear correlation was observed between the concentration of airborne fungi and meteorological elements. For Seonamsa temple, on the other hand, a strong correlation was found between the concentration of airborne fungi and number of days with precipitation, relative humidity, precipitation, and total horizontal radiation. In light of the meteorological characteristics, this difference may be attributed to Beopjusa temple having a higher mean wind speed, total horizontal radiation, and total evaporation, and to Seonamsa temple having higher mean air temperature, mean relative humidity, mean dew point temperature, precipitation, and number of days with 
precipitation. It has been reported that the meteorological characteristics of Seonamsa temple provide more favorable conditions for microbial growth(Kim et al., 2017). and Further, the results of this study also provide evidence for the observed correlation where the concentration of airborne fungi is higher during the season of larger precipitation and higher in Seonamsa temple, where the amount of precipitation is greater. Thus, at Seonamsa temple, the linear correlation between meteorological elements and the concentration of airborne fungi is high. However, the findings in this study were in contrast to the report by Wang et al.(2010) where the concentration of airborne fungi showed strong positive correlations with air temperature and the number of visitors, but strong negative correlations with relative humidity and precipitation. The results obtained by Wang et al.(2010) seems to be due to a greater consideration of an artificial cause such as the number of visitors(Wang et al., 2010) than of the influence of topography(De Linares et al., 2010), region, and season(Medrela-Kuder, 2003; Oliveira et al., 2005), wind, air temperature, relative humidity, precipitation, elevation, and vegetation of the surrounding areas(Topbas et al.,
2006). Generally, the concentration of airborne fungi shows strong positive correlations with humidity and precipitation. In addition, the results of the correlation analysis, including the number of visitors on the days of sampling, showed that there no linear correlations between the concentration of airborne fungi and the number of visitors in both Beopjusa and Seonamsa temples. In addition, the correlation analysis differed between the Beopjusa and Seonamsa temples on the basis of the common and individual properties. Common properties have consistently strong linear correlations with the parameters irrespective of topography, geography, or other conditions, while the individual properties have, as shown in Figure 2(Lee, 2015), strong linear correlations with the meteorological characteristics of the Beopjusa and Seonamsa temples based on the topographical conditions including altitude, relative height, relative relief, slope, topographic direction, direction of open terrain, and distance from the coast. The result agreed with the study where topographical conditions at the monitoring sites were highlighted as the reason for the greater influence of the change in monitoring sites on humidity, wind speed, and

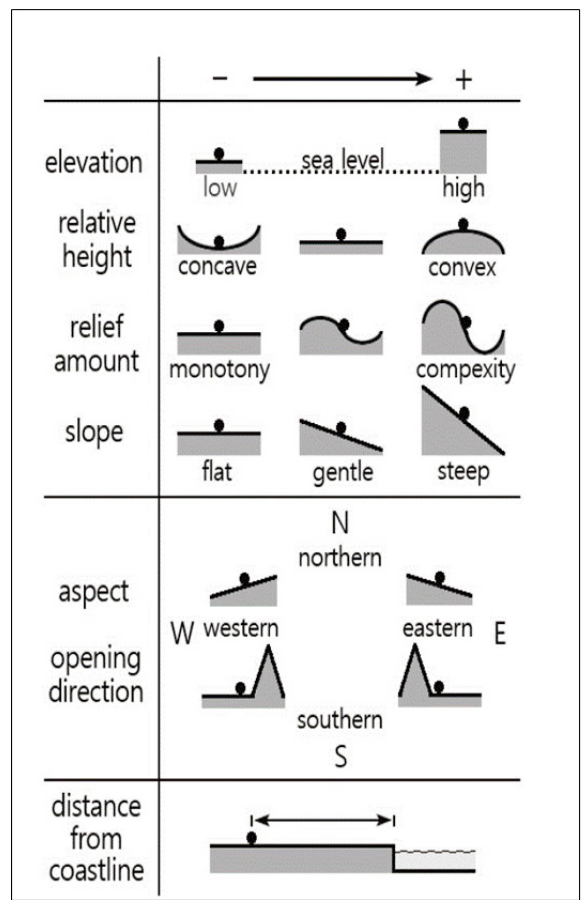

\begin{tabular}{|c|c|}
\hline $\begin{array}{c}\text { Beopjusa } \\
\text { Temple }\end{array}$ & $\begin{array}{c}\text { Seonasa } \\
\text { Temple }\end{array}$ \\
\hline high & high \\
concave & concave \\
\hline $\begin{array}{c}\text { monotony } \\
\text { flat }\end{array}$ & $\begin{array}{c}\text { compexity } \\
\text { steep }\end{array}$ \\
\hline $\begin{array}{c}\text { southern } \\
\text { (SSW) }\end{array}$ & $\begin{array}{c}\text { southern } \\
\text { (SSE) }\end{array}$ \\
\hline $\begin{array}{c}\text { southern } \\
\text { (SSW) }\end{array}$ & $\begin{array}{c}\text { southern } \\
\text { (SSE) }\end{array}$ \\
\hline $\begin{array}{c}\text { (120 km) } \\
\text { middle } \\
\text { (20 km) }\end{array}$ \\
\hline
\end{tabular}

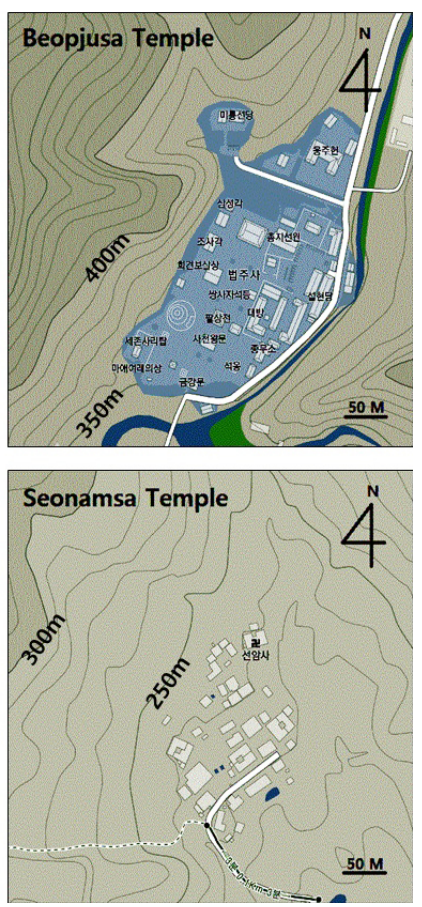

Figure 2. Topographical condition of the Beopjusa and Seonamsa temples(Lee, 2015). 
wind direction than on air temperature and sea level pressure (Meteorological Research Institute Korea Meteorological Administration, 2006). The unique meteorological characteristics of the Beopjusa and Seonamsa temples are thought to have come from the individual topographical conditions of their sites, where they are similar with respect to altitude, relative height, topographic direction, and direction of open terrain, but differ in terms of relative relief, slope, and distance from the coast(Figure 2).

Based on the above results and discussion, the following conclusions have been drawn:

1. Beopjusa temple exhibits higher values for wind speed, total horizontal radiation, evaporation, and number of days with precipitation, while Seonamsa temple exhibits higher values for air temperature, relative humidity, dew point temperature, sea level pressure, precipitation, and concentration of airborne fungi. The higher concentration of airborne fungi was correlated with higher precipitation. The highest concentrations were observed in summer, followed by fall, in Beopjusa temple, whereas in Seonamsa temple, the highest concentrations were observed in spring, followed by summer.

2. Pearson correlation between meteorological elements and the concentration of airborne fungi showed that, for Beopjusa temple, no linear correlation was observed between the concentration of airborne fungi and meteorological elements, while for Seonamsa temple, the concentration of airborne fungi had strong correlations with number of days with precipitation, relative humidity, precipitation, and total horizontal radiation. Furthermore, the correlations were differentiated according to the common characteristics of the Beopjusa and Seonamsa temples, where they shared correlations unaffected by topographical conditions, and the individual characteristics, where the two temples had independent correlations under the influence of their unique topographical conditions.

\section{Acknowledgement}

This study was funded by the 2015, 2016, and 2017 Cultural Heritage R\&D Project of the National Research
Institute of Cultural Heritage, Cultural Heritage Administration, and we are deeply grateful for their administrative and financial support.

\section{REFERENCES}

Abdel Hameed, A.A., Khoder, M.I., Ibrahim, Y.H., Saeed, Y., Osman, M.E. and Ghanem, S., 2012, Study on some factors affecting survivability of airborne fungi. Science of the Total Environment, 414, 696-700.

Alghamdi, M.A., Shamy, M., Redal, M.A., Khoder, M., Awad, A.H. and Elserougy S., 2014, Microorganisms associated particulate matter: A preliminary study. Science of the Total Environment, 479-480, 109-116.

De Linares, C., Belmonte, J. and Canela, M., 2010, Dispersal patterns of alternaria conidia in Spain. Agr Forest Meteorol, 150, 1491-1500.

Hong, J.Y., Kim, Y.H., Lee, J.M., Jo, C.W. and Park, J.H., 2018, Seasonal distribution and diversity of airborne fungi in a wooden cultural heritage site: A case study of the Seonamsa temple, Suncheon. The Korean Journal of Mycology, 46(2), 122-133. (in Korean with English abstract)

Jogye Order of Korean Buddhism - Beopjusa Temple, 2018, Cultural heritage guide. http://www.beopjusa.org/ (June 22, 2018)

Kim, M.N., Lim, B.A., Lee, M.S. and Jeong, S.Y., 2017, Comparison of characteristics of local meteorological and particulate matter(TSP) on the Beopjusa temple and Seonamsa temple. Journal of Conservation Science, 33(4), 283-295. (in Korean with English abstract)

Lee, G.R., 2015, Relationships between topographical conditions and climatic data at weather stations in South Korea. Climate Research, 10(3), 247-262. (in Korean with English abstract)

Medrela-Kuder, E., 2003, Seasonal variations in the occurrence of culturable airborne fungi in outdoor and indoor air in Cracow. Int Biodeterior Biodegradation, 52, 203-205.

Meteorological Research Institute Korea Meteorological Administration, 2006, Diagnosis of characteristics of local meteorology and development of techniques for the meteorological environmental impact assessment (I). 
1-218. (in Korean with English abstract)

National Research Institute of Cultural Heritage, 2011, Cleaning and Surface Contaminant of Stone Cultural Heritage. 7. (in Korean)

National Research Institute of Cultural Heritage, 2014, Conservation management of stone cultural heritage using nondestructive technology. 13, 15.(in Korean)

Nilsson, T. and Rowell, R., 2012, Historical wood - structure and properties. Journal of Cultural Heritage. 13S, S5-S9.

Oliveira, M., Ribeiro, H. and Abreu, I., 2005, Annual variation of fungal spores in atmosphere of Porto: 2003. Ann Agric Environ Med, 12, 309-315.

Southworth, D., 1973, Introduction to the biology of airborne fungal spores. Ann Allergy, 32, 1-22.

Taego Order of Korean Buddhism - Seonamsa Temple, 2018, Cultural heritage guide. http://www.seonamsa.net/ (June $22,2018)$

Topbas, M., Tosun, I., Can, G., Kaklikkaya, N. and Aydin, F., 2006, Identification and seasonal distribution of airborne fungi in urban outdoor air in an eastern Black Sea Turkish town. Turk J Med Sci., 36, 31-36.

Wang, W., Ma, X., Ma, Y., Mao, L., Wu, F., Ma, X., An, L. and Feng, H., 2010, Seasonal dynamics of airborne fungi in different caves of the Mogao Grottoes, Dunhuang, China. International Biodeterioration \& Biodegradation, 64, $461-466$ 